\title{
La comunicación de la identidad de la marca Gucci en su perfil de Instagram
}

\section{The communication of Gucci's brand identity on its Instagram profile}
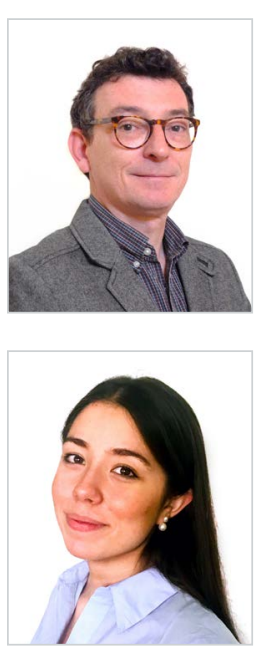

Recibido: 15/05/2021 - Aceptado: 03/11/2021 - En edición: 10/12/2021 - Publicado: 01/01/2022

\section{Resumen:}

En la actualidad, los perfiles de las marcas de moda en Instagram son seguidos por una amplia comunidad de jóvenes usuarios. Una de las firmas más destacadas en la red social es Gucci, cuya estrategia de comunicación digital ejemplifica los esfuerzos de algunas marcas de lujo por reorientarse hacia los nuevos perfiles de consumidores. El principal objetivo de este trabajo es conocer de qué manera comunica Gucci su identidad de marca en su perfil de Instagram durante el periodo de lanzamiento de una de sus colecciones. Mediante un análisis de contenido cuantitativo sobre una muestra de 642 publicaciones, se registran las asociaciones de marca estructuradas en torno a las dimensiones de producto, organización, persona y símbolo, según el modelo de identidad de marca formulado por Aaker (1996). Los resultados
Received: 15/05/2021 - Accepted: 03/11/2021 - Early access: 10/12/2021 - Published: 01/01/2022

\section{Abstract:}

Nowadays, a large community of young users follow the profiles of fashion brands on Instagram. One of the most prominent companies on this social network is Gucci, whose digital communication strategy exemplifies the endeavour of some luxury brands to redirect their efforts toward new consumer profiles. The purpose of this study is to determine the way in which Gucci communicated its brand identity on its Instagram profile during the launch of one of its collections. By using quantitative content analysis with a sample of 642 publications, brand associations structured around the aspects of product, organisation, person, and symbol have been registered according to the brand identity model formulated by Aaker (1996). The results show that the content of the publications is consistent with a brand identity that presents itself as

Cómo citar este artículo:

Carcavilla Puey, F., Aguirre Jiménez, A. I. (2022). La comunicación de la identidad de la marca Gucci en su perfil de Instagram. Doxa Comunicación, 34, pp. 177-200. 
muestran que los contenidos de las publicaciones son coherentes con la identidad de una marca que se presenta como ecléctica, moderna, romántica, exclusiva e influyente. Estos atributos se manifiestan principalmente en los productos de la marca y en la figura de su director creativo.

\section{Palabras clave:}

Moda de lujo; identidad de marca; redes sociales; Instagram; Gucci.

\section{Introducción}

El dinamismo de la moda es un reflejo de la evolución de los gustos y tendencias de la sociedad. Esto se pone de manifiesto en la constante renovación de sus propuestas y en la forma de comunicarlas a las nuevas generaciones de consumidores.

En la actualidad, las redes sociales han adquirido un destacado protagonismo en la comunicación de las empresas del sector de la moda y, en particular, en el ámbito del lujo (Estévez-Martín et al., 2019). Entre ellas, Instagram resulta especialmente atractiva para las marcas por su carácter visual y su capacidad para generar engagement entre sus usuarios (Oliveira y Fernandes, 2020; Vicente-Fernández et al., 2020; Moreno, 2018).

Otra de sus ventajas es la elevada tasa de crecimiento de su audiencia (Estévez-Martín et al., 2019; Ávila, 2017), cuyo perfil mayoritario corresponde a personas de entre 25 y 34 años (Statista, 2021a). De este modo, Instagram se ha convertido en la plataforma de referencia para los millennials, "la primera generación que cambia por completo sus hábitos de consumo y estilo de vida respecto a la anterior" (Pérez-Curiel y Sanz-Marcos, 2019: 4).

De entre las firmas de moda presentes en Instagram, Gucci ha sido seleccionada para el presente estudio por su popularidad y por su valor diferencial en la comunicación digital con las nuevas audiencias (Sanz-Marcos y Pérez-Curiel, 2019).

El ranking elaborado por Interbrand Best Global Brands (2020) sitúa a Gucci como la cuarta marca mejor valorada en el sector del lujo en el año 2020, por detrás de Louis Vuitton, Chanel y Hermès. Asimismo, la firma italiana ocupa el tercer puesto entre las marcas de moda más populares en 2020, por detrás de Nike y Off-White según el estudio realizado por The Lyst Index (2020).

El creciente protagonismo de Instagram en la comunicación digital de las marcas de moda y la relevancia de Gucci como paradigma de marca de lujo reorientada hacia las nuevas generaciones de consumidores dirigen nuestro interés a la comunicación que la firma italiana realiza en dicha plataforma. En concreto, nuestro propósito es conocer de qué manera comunica Gucci su identidad de marca en su perfil de Instagram durante el periodo de lanzamiento de una de sus colecciones.

Este objetivo principal, se concreta en los siguientes objetivos específicos:

- Detectar los principales atributos de identidad de la marca Gucci.

- Describir cómo se plasma la identidad de la marca en Instagram a través de sus dimensiones como producto, organización, persona y símbolo.

- Determinar si existe una coherencia entre los contenidos publicados por la marca y los principales atributos con los que esta se identifica. 


\section{Estado de la cuestión}

\subsection{La comunicación de la moda en Instagram}

Los cambios en la economía global y los avances en las tecnologías de la comunicación han conducido a notables transformaciones en las demandas y hábitos de consumo de la nueva generación de consumidores de marcas de moda de lujo (Ko et al., 2016; Alonso y Arébalos, 2011). Este nuevo perfil de consumidor está acostumbrado a "explorar, comparar, compartir y comprar por Internet aquellas marcas por las que se siente atraído" (Pérez-Curiel y Sanz-Marcos, 2019: 7). Esto exige la búsqueda de fórmulas de comunicación menos intrusivas que la publicidad tradicional (Liberos et al., 2013).

Las redes sociales son estructuras compuestas por personas que comparten intereses comunes o algún tipo de relación a través de Internet (Iab Spain, 2021). Todas tienen en común ser una red de contactos, permitir tener un perfil de usuario, y ofrecer herramientas para interaccionar con contenidos (crear, compartir y/o participar). A este respecto, se pueden definir como "un espacio virtual para compartir información y reanudar o comenzar conversaciones" (Llamas y Pagador, 2014: 45).

La comunicación en redes sociales permite crear una comunidad entre las marcas y sus usuarios, principalmente los más jóvenes. Estos, a su vez, se convierten en generadores de contenidos vinculados a la marca (Estévez-Martín et al., 2019). El concepto de social media marketing hace referencia a aquellas comunicaciones de marketing en plataformas digitales que facilitan la interacción, la colaboración y los contenidos compartidos entre los usuarios y las marcas (Kim y Ko, 2012). En ese sentido, las redes sociales resultan atractivas para las marcas por su elevada capacidad de segmentación y la facilidad de medición de las acciones, lo que permite optimizar la afinidad de los contenidos con el público (Estévez-Martín et al., 2019).

En un principio, las marcas de moda de lujo se mostraron reticentes a utilizar las redes sociales debido a su naturaleza inclusiva y accesible, totalmente opuesta al concepto de lujo (Park et al., 2019). En la actualidad, afrontan el reto de mantener la percepción de exclusividad mientras permiten el amplio acceso a las masas (Oliveira y Fernandes, 2020; Pentina et al., 2018).

No obstante, la pandemia de COVID-19 ha acelerado la adopción de nuevas tecnologías en el sector del lujo para compensar el cierre de las tiendas físicas y la consecuente disminución de puntos de contacto con el cliente (Deloitte, 2020). A este respecto, cabe señalar que la industria de la moda incrementó un $52 \%$ su inversión en redes sociales durante el primer trimestre de 2021 respecto al mismo periodo de 2020 (Socialbakers, 2021).

Una de las plataformas más populares en la actualidad es Instagram, cuyo atractivo para las marcas reside, entre otras razones, en su naturaleza visual y su elevada tasa de crecimiento. En la actualidad, desempeña un papel fundamental en las estrategias de comunicación de las marcas de moda de lujo (Estévez-Martín et al., 2019; Ávila, 2017). Como señalan Oliveira y Fernandes (2020), las personas que interactúan con una marca de moda de lujo en Instagram son más propensas a tener una mejor imagen y mostrar más fidelidad a la marca.

Instagram fue concebida en 2010 como una red para compartir fotografías, aunque no tardó en incluir la opción de compartir vídeos (Moreno, 2018). Recientemente, ha aumentado las posibilidades de generar contenido al incorporar herramientas como Instagram TV (vídeos de una duración superior a los que se pueden compartir únicamente en el muro de la plataforma), Reels (vídeos de hasta 60 segundos) o Instagram Stories (vídeos o fotografías de hasta 15 segundos de duración que permanecen públi- 
cos 24 horas). Estas nuevas funcionalidades de la aplicación permiten a las marcas desarrollar estrategias de storytelling que se desarrollan en el tiempo y generan un mayor engagement con los usuarios (Estévez-Martín et al., 2019).

Son varios los autores que destacan la idoneidad de Instagram sobre otras plataformas para generar engagement (Oliveira y Fernandes, 2020; Vicente-Fernández et al., 2020; Moreno, 2018), por lo que vamos a desarrollar brevemente este concepto. Según Dessart et al. (2015), el engagement consiste en establecer relaciones interactivas entre el consumidor y la marca. En el caso de Instagram, las interacciones se calculan sumando los likes y comentarios que reciben las publicaciones de un perfil. En el primer trimestre de 2021, el número de interacciones en dicha plataforma fue 22,7 veces superior a las de Facebook (Socialbakers, 2021). Sin embargo, Velar-Lera et al. (2020) consideran que las marcas de moda de lujo no aprovechan todo su potencial para estimular la participación de los usuarios y generar comunidad.

Otra de las ventajas de Instagram es el potencial de crecimiento de su audiencia, cuya cifra se estima actualmente en 1074 millones de usuarios activos mensuales a nivel mundial (Statista, 2021b). Su tasa interanual de crecimiento durante el primer trimestre de 2021 fue de un 10,3\%, mientras que Facebook apenas obtuvo variación durante el mismo periodo, al aumentar un 0,8\% (Socialbakers, 2021).

En cuanto al perfil de usuario de la plataforma, Instagram tiene un mayor grado de afinidad entre mujeres (51,4\%) que entre hombres (48,6\%) (Statista 2021c) y su nivel de penetración más elevado lo alcanza en la población de entre 25 y 34 años, con un 32,1\% (Statista, 2021a). Además, algo más de dos tercios de los usuarios de Instagram tienen menos de 35 años (Statista, 2021 a). Estos datos también suponen un incentivo para las marcas de moda de lujo, cuya supervivencia está necesariamente unida al rejuvenecimiento de su público (Pérez-Curiel y Sanz-Marcos, 2019).

\subsection{La construcción de la identidad de la marca}

Las marcas son las principales protagonistas del sector de la moda, donde no solo actúan como elemento distintivo del fabricante, sino que ejercen una función simbólica y trasladan al cliente una proposición de valor única y diferenciada. Como señala Okonkwo (2009), el lujo asociado a las marcas se caracteriza por un estilo reconocible, una identidad fuerte, un alto reconocimiento y fuertes asociaciones emocionales y simbólicas.

La imagen de marca se vincula a los públicos, al tratarse del conjunto de asociaciones mentales que estos tienen de la marca, producto u organización. Por otra parte, la identidad de marca se vincula a la organización, dado que se compone de un conjunto de atributos o características centrales, distintivas y perdurables con las que esta se identifica y diferencia de sus competidores (Capriotti, 2009).

Aaker (1996) propone abordar la identidad de marca desde cuatro perspectivas: producto, organización, persona y símbolo. De este modo, se pretende definir los atributos o asociaciones mentales que se van a vincular a la marca para articular su significado. A continuación, vamos a desarrollar estas cuatro dimensiones, dado que posteriormente las utilizaremos como referencia para estructurar nuestro análisis.

En primer lugar, la perspectiva de producto contempla las asociaciones de marca vinculadas a las características del producto o categoría de productos que comercializa. Aaker (1996) señala las limitaciones de esta perspectiva, dado que se basa principal- 
mente en atributos funcionales de los productos, asumiendo una excesiva racionalidad en la toma de decisiones del cliente. Por lo tanto, el autor recomienda ampliar las perspectivas de identidad de la marca más allá del producto.

No obstante, el mercado de la moda de lujo se compone de marcas aspiracionales con un marcado carácter simbólico (Okonkwo, 2009). Esto implica que las decisiones de compra tienen un elevado componente emocional. El producto materializa y concentra la esencia de la marca y provoca una reacción emocional en el consumidor al despertar en su mente una serie de asociaciones con los atributos y valores que esta representa (Girón, 2011). La singularidad y exclusividad de la marca se plasman en la alta calidad del producto, su distribución exclusiva y su elevado precio (Okonkwo, 2009).

La perspectiva de marca como organización trasciende las asociaciones con productos específicos y se orienta a potenciar las características singulares de la organización que los respalda. Las asociaciones de marca basadas en los valores de la compañía, su cultura corporativa, las personas que la integran o su responsabilidad social, son más perdurables y se transfieren a todos sus productos. En el ámbito que nos ocupa, algunas marcas de moda de lujo se vinculan al arte y la cultura mediante fórmulas como el patrocinio y el mecenazgo y, de este modo, enriquecen su relato (Velar-Lera et al., 2020). En consecuencia, Aaker (1996) considera que esta perspectiva es más completa y recomendable que la anterior.

El desarrollo de la personalidad de marca es la tercera perspectiva desde la que trabajar su identidad. Según Aaker (1996), dotar de características humanas a una marca favorece que los consumidores se identifiquen con ella, permite guiar sus acciones de comunicación y contribuye a la diferenciación respecto a sus competidores. La personalidad de la marca se puede consolidar estableciendo vínculos con personas que representen características y valores atribuibles a la marca.

En primer lugar, la personalidad y los valores asociados al fundador de la marca trazan las líneas maestras en las que se asienta su identidad (Capriotti, 2009). Un claro ejemplo en el ámbito de la moda es la influencia que la figura de Coco Chanel sigue ejerciendo sobre la firma francesa tras más de un siglo de existencia (Gautier, 2011). Asimismo, el director creativo resulta esencial en la construcción de la personalidad de la marca de moda (Velar-Lera et al., 2020).

Con frecuencia, las marcas de moda de lujo recurren en sus estrategias de promoción al celebrity endorsement o respaldo de celebridades. El "capital de celebridad”, o notoriedad adquirida por estas personas mediante la acumulación de visibilidad mediática, permite a las marcas alcanzar a sectores del público más amplios (Driessens, 2013). Para establecer estas asociaciones, las marcas utilizan diversas fórmulas como la publicidad convencional, el emplazamiento de producto en cine y televisión o la presencia de celebridades en eventos vistiendo ropa y accesorios de la marca (Estévez-Martín et al., 2019; Okonkwo, 2007).

En el ámbito de la moda de lujo, Barron (2021) utiliza el concepto de "musa" para referirse a aquellas celebridades cuyo rol no se limita a ceder su imagen para las campañas publicitarias de la marca, sino que encarnan su identidad e inspiran a sus diseñadores. Según el autor, esta figura representa la máxima integración de la celebridad con la comunicación de la marca de moda.

En la actualidad, los modelos profesionales mantienen su protagonismo en la pasarela, pero están siendo desplazados por las celebridades como cara visible de las marcas. De este modo, se ha desdibujado la línea que separa a los modelos de las celebridades (Barron, 2021). De cualquier forma, las características y rasgos de los modelos escogidos para las colecciones son otro factor clave para mostrar la personalidad de la marca. 
El creciente protagonismo adquirido por los influencers en la comunicación de moda requiere que nos detengamos en las características de esta figura. Según Vicente-Fernández et al. (2020), los influencers o prescriptores digitales son un nuevo modelo de líder de opinión con capacidad para influir en las actitudes y conductas de consumo de determinados colectivos. Por esta razón, son elegidos por las marcas como embajadores e intermediarios con sus públicos en sus estrategias de marketing.

El influencer nativo ha adquirido su fama en las redes sociales (Iab Spain, 2019) y utiliza estas plataformas para impulsar su perfil y construir su propia marca personal (Carrillat e Ilicic, 2019; Pérez-Curiel y Sanz-Marcos, 2019; Fernández-Gómez et al., 2018). En ocasiones, el volumen de seguidores de su cuenta de Instagram supera con creces la cifra del perfil oficial de las marcas de moda (Amed, 2015). En ese sentido, los influencers pueden clasificarse en función de su número de seguidores. Por ejemplo, se considera que un microinfluencer en Instagram se sitúa entre los 10.000 y los 50.000 seguidores y un macroinfluencer oscila entre los 250.000 y el millón (Iab Spain, 2019).

Por último, Aaker (1996) plantea la perspectiva de la marca como símbolo, que alude a su imaginario visual y herencia histórica. Toda representación física de la marca puede ejercer una función simbólica cuando es reconocible y memorable por el público, constituyendo un valioso activo para la compañía.

\subsection{Gucci como paradigma de marca de lujo}

Influyente, innovadora y vanguardista. Estos son los tres adjetivos con los que se presenta la icónica firma tras cien años de historia (Gucci, s.f.).

En 1921 nació una nueva marca: 'Valgeria Gucci', que se convertiría tiempo después en una de las mayores firmas de la alta costura. Comenzó de la mano de su fundador, Guccio Gucci, con los artículos de cuero como su principal producto (Jackson y Haid, 2002).

Los años cuarenta supusieron un gran cambio para la firma y la concepción que el mundo tenía de la misma. Del pequeño taller, Gucci pasó a ser reconocida internacionalmente al término de la Segunda Guerra Mundial (Yoffie y Kwak, 2001). Con el paso del tiempo, la firma aumentó su presencia dentro del mundo de la moda. En 1953 llegó a Nueva York con la apertura de su primera tienda situada fuera de Italia. Según indican los autores, "aquellos fueron los años dorados de Gucci" (2001: 2).

En 1970, los hijos de Guccio, Aldo y Rodolfo, se convirtieron en socios igualitarios de la compañía. Este cambio derivó en una falta de liderazgo y una pérdida de visión estratégica. El lanzamiento en 1979 de la nueva línea GAC (Gucci Accessories Collection), una extensión de marca creada para alcanzar a públicos más amplios, supuso una pérdida de control sobre la calidad. La amplia concesión de licencias afectó a la percepción de exclusividad de la marca, situación que empeoró con la proliferación de falsificaciones (Jackson y Haid, 2002).

Cuando Maurizio, el nieto de Guccio, tomó las riendas en 1984, tuvo que hacer frente a la crisis de la compañía. A su llegada, recortó la plantilla y eliminó la concesión de licencias, acotó el número de establecimientos, se hizo con las franquicias de Norteamérica y amplió el control sobre la distribución, pasando a ser una gestión interna (Jackson y Haid, 2002).

Asimismo, Domenico De Sole fue nombrado presidente y director gerente de Gucci América y Dawn Mello fue elegida como directora creativa. Con su llegada, Gucci cambió su forma de comprender la moda (Vogue, s.f.). La firma rechazó la innovación y 
volvió la vista a sus orígenes. Alejada de las tendencias, Mello recuperó el bolso de asas de bambú, se deshizo de gran parte de la producción y modificó algunos diseños clásicos como los mocasines horsebit (Yoffie y Kwak, 2001).

La gran sucesión de cambios dentro de la firma terminó por afectar a la economía: "los precios eran demasiado altos, la producción estaba desorganizada y el abastecimiento era una pesadilla" (Yoffie y Kwak, 2001: 4).

Ante esta situación, Maurizio vendió en 1993 Gucci al grupo de inversión Investcorp (Vogue, s.f.). Tras este suceso, se produjo una reorganización en los roles de la empresa, siendo sustituido Dawn Mello por Tom Ford en 1994, quien renovó la imagen de la marca clásica, hasta entonces enfocada a personas con un mayor rango de edad, abriendo paso a una nueva consumidora de Gucci más joven y moderna (Yoffie y Kwak, 2001). Además, De Sole bajó los precios un 30\% y se incrementó el gasto en comunicación y publicidad.

Ford y De Sole abandonaron la firma en el año 2004, tras la compra de Gucci por el Grupo Kering, del cual sigue formando parte en la actualidad. Dentro del grupo, la firma italiana comparte espacio con otras marcas del sector de la moda de lujo, pero sin duda, Gucci ocupa un papel fundamental, suponiendo el 63\% de los beneficios de la agrupación (Martínez, 2020).

Un estudio realizado por Deloitte (2019) hace una radiografía del mercado internacional del lujo a partir del análisis de las cien marcas que generan más ingresos. El grupo Kering se encuentra en cuarta posición en ventas, con unos ingresos totales de 17.446 millones de dólares en el año 2017 y un crecimiento del 27,5\% respecto al ejercicio anterior. Esto supone que el grupo empresarial se sitúe en octavo lugar entre las 20 compañías que experimentaron un crecimiento más rápido entre los años 2015 y 2017 (Deloitte, 2019).

El crecimiento de la marca coincide con la incorporación de Alessandro Michele como director creativo de Gucci en 2015. La llegada del diseñador ha supuesto una revolución para la firma italiana:

Tras la mirada del nuevo director creativo Alessandro Michele, la Maison ha redefinido el lujo en el siglo XXI, reforzando aún más su posición como una de las marcas de moda más deseadas del mundo. Eclécticos, modernos y románticos, los productos Gucci representan la cumbre de la artesanía italiana gracias a su insuperable nivel de calidad y meticulosidad (Gucci, s.f.).

El eclecticismo de Alessandro Michele se ve reflejado en la introducción de nuevos iconos como serpientes, tigres y elementos florales que comparten protagonismo con la clásica franja bicolor. No obstante, el diseñador ha recuperado algunos símbolos del pasado que permanecían en desuso, como el monograma de la doble G en honor al fundador de la marca Guccio Gucci. Según Michele, hubo un tiempo en el que la firma se avergonzó de su símbolo, algo que, en su opinión, debería suscitar todo lo contrario, un orgullo por un emblema de tantos años (The Fashion Law, 2016).

La llegada de Alessandro Michele también ha supuesto un importante avance en la comunicación digital de la marca. En colaboración con Marco Bizarri, CEO de la compañía, el director creativo sentó las bases de un plan estratégico en 2017 centrado en la interacción con un público más joven. En consecuencia, las plataformas digitales, y en especial, Instagram, adquieren un mayor protagonismo en la comunicación de la marca (Pérez-Curiel y Sanz-Marcos, 2019).

Según Velar-Lera et al. (2020), Gucci destaca sobre otras marcas de moda de lujo por su elevada actividad en Instagram, al registrar una media de 3,7 posts diarios. Asimismo, la marca renueva su estética y su lenguaje orientándose al nuevo perfil de público 
millennial. Estos cambios "marcan la revolución digital que convierte a Gucci en la marca más popular y visualizada en los portales de Internet” (Pérez-Curiel y Sanz-Marcos, 2019: 7).

Sin embargo, Pérez-Curiel y Sanz-Marcos (2019) demuestran que Gucci hace un uso moderado de los influencers en su comunicación en Instagram, reforzando su independencia de agentes externos. En la nueva estrategia de comunicación de Gucci, la figura de Michele ocupa un lugar preferente al posicionarse como influencer desde la cuenta oficial de la marca. Asimismo, el producto se sitúa como el elemento central de la comunicación y principal activo de la marca, la cual se posiciona "como líder de opinión legítimo e influyente para su comunidad de seguidores" (Pérez-Curiel y Sanz-Marcos, 2019: 19).

Para definir la identidad de Gucci, es necesario conocer aquellas características centrales, distintivas y perdurables con las que la marca se identifica y diferencia de sus competidores (Capriotti, 2009). A partir de la información expuesta anteriormente, hemos detectado una serie de atributos que Gucci comunica de forma explícita. Para mejorar su comprensión, los hemos agrupado en tres ejes siguiendo un criterio de temporalidad, tal como se muestra en la tabla 1.

Tabla 1. Atributos de identidad de Gucci

\begin{tabular}{|l|l|l|}
\hline Pasado & Presente & Futuro \\
\hline $\begin{array}{l}\text { Romántica } \\
\text { De calidad } \\
\text { Artesanía italiana }\end{array}$ & $\begin{array}{l}\text { Moderna } \\
\text { Ecléctica } \\
\text { Exclusiva }\end{array}$ & Influyente \\
\hline
\end{tabular}

Fuente: elaboración propia

En primer lugar, encontramos atributos referidos al heritage que la marca ha acumulado tras un siglo de historia. Gucci se identifica con la tradición artesanal y el saber hacer que entroncan directamente con sus orígenes. Estos atributos confieren legitimidad y autenticidad a la marca. Hemos incluido el atributo "romántica" en este eje por su connotación de nostalgia e idealización del pasado. Sin embargo, se trata de un concepto muy amplio que puede abarcar diversos significados como rebelde, transgresora, fantástica, idealista, independiente, libre o pasional.

El eje del presente establece un nexo entre la tradición y la vanguardia. La marca se identifica como moderna o perteneciente al tiempo actual. Asimismo, se define como ecléctica, es decir, que combina distintos estilos y elementos existentes para obtener un resultado original y novedoso. El término ecléctico puede tener connotaciones de extravagante o poco común. Además, enlaza con el romanticismo en cuanto a la transgresión de la norma y el rescate de elementos del pasado, cuya reinterpretación es una actitud propia de la postmodernidad (Campi, 2007). El eclecticismo también nos conduce a la idea de diversidad frente a lo homogéneo. Por último, si bien Gucci no menciona explícitamente el atributo de "exclusiva", este se desprende de la alineación de la marca con el concepto de lujo y de su identificación como "una de las marcas de moda más deseadas del mundo" (Gucci, s.f.).

Por último, situamos los atributos de influyente, innovadora y vanguardista en el eje del futuro. De este modo, Gucci se posiciona a la vanguardia de la moda como una marca creativa que asienta las bases de las futuras tendencias, destacando su rol de influencia como señalan Pérez-Curiel y Sanz-Marcos (2019). 


\section{Objetivos e hipótesis}

Lo expuesto anteriormente explica el atractivo de Instagram como plataforma de comunicación para las marcas de moda, tanto por su naturaleza visual e interactiva como por las características de sus usuarios. Asimismo, comprobamos que Gucci es una marca con un amplio recorrido en el sector del lujo cuyo reto permanente es renovarse sin perder su esencia para conectar con las nuevas generaciones de consumidores. La redefinición llevada a cabo por Alessandro Michele supone un cambio en la proposición de valor de la marca y, consecuentemente, en su estilo de comunicación. Así, el propósito general de esta investigación es estudiar la comunicación de la identidad de la marca Gucci a través de su perfil de Instagram durante el lanzamiento de una de sus colecciones.

De una manera más específica, queremos detectar los principales atributos de identidad de la marca Gucci. Asimismo, es nuestro propósito concretar cómo se plasma la identidad de la marca en Instagram a través de sus dimensiones como producto, organización, persona y símbolo. Finalmente, queremos determinar si existe una coherencia entre los contenidos publicados por la marca y los principales atributos con los que esta se identifica.

En virtud de los objetivos, y a partir de la revisión literaria realizada, se plantean las siguientes hipótesis de investigación:

H1: La figura del director creativo de Gucci, Alessandro Michele, ocupa un lugar preferente en las publicaciones de la marca respecto a otras celebridades e influencers, cuyo protagonismo es inferior.

H2: El eclecticismo de la marca no solo queda reflejado en sus diseños, sino también en la diversidad y características físicas de sus modelos.

H3: La marca muestra una elevada vinculación con sus productos en los contenidos de sus publicaciones.

H4: La comunicación de Gucci en Instagram es coherente con los principales atributos de identidad de la marca.

\section{Metodología}

Para analizar la comunicación de la firma italiana en Instagram, se ha llevado a cabo un análisis de contenido de corte cuantitativo entendido como: "la técnica sin duda más elaborada y que goza de mayor prestigio científico en el campo de la observación documental" (Sierra, 1997: 287). Este método, que permite descubrir el contenido manifiesto de la comunicación de una manera sistemática, objetiva y cuantitativa (Berelson, 1952), tiene como fin medir unas variables concretas (Kerlinger, 1986).

El carácter sistemático del análisis supone calcular la frecuencia con la que se manifiestan ciertos rasgos en un contenido, según señala Zurutuza (2012). Para ello, el investigador deberá delimitar el objeto de estudio, la muestra, la franja temporal y las unidades de análisis. Una vez establecidos, será necesario que los procesos de codificación, análisis y evaluación estén uniformados (Wimmer y Dominick, 1996). La delimitación de los procesos buscará la difícil objetividad del análisis de contenido, al no poder influir la opinión del investigador en los resultados.

En la presente investigación, el objeto de estudio es la comunicación de Gucci en su cuenta oficial de Instagram @gucci durante el lanzamiento de una de sus colecciones. Para ello, la muestra seleccionada han sido las publicaciones realizadas desde el 27 de 
septiembre de 2019 al 12 de enero de 2020. Esta delimitación temporal responde al periodo que abarca desde la primera publicación referente a la colección primavera-verano 2020, hasta el anuncio de la colección otoño-invierno.

Definimos como unidad codificable cada una de las publicaciones que conforman el muro principal de la firma italiana. Se decidió estudiar únicamente este contenido al ser la primera página que se visualiza al entrar en el perfil de Gucci. Respecto al contenido, cabe apuntar que, en el caso de las galerías, cada uno de los elementos que las componen serán considerados unidades independientes. Por otro lado, quedan excluidos de la codificación tanto los Stories, los filtros, los Instagram TV que no fueron publicados en el muro, así como las imágenes etiquetadas y los Reels, ya que estos últimos no existían al inicio de la investigación, por lo que no se contemplaron a la hora de elaborar el código. De esta manera, obtuvimos un total de 642 unidades de análisis.

El diseño del código es original y su estructura se fundamenta en las cuatro perspectivas de la identidad de marca formuladas por Aaker (1996). El código comprende veintitrés variables con sus respectivos valores, tal como se muestra en la tabla 2 . Al término de la investigación, los datos fueron tratados con el programa SPSS (Statistical Package for the Social Sciences) y se muestran como tablas de frecuencia en el siguiente apartado.

Tabla 2. Categorías de análisis empleadas en la investigación

\begin{tabular}{|c|c|c|}
\hline \multicolumn{3}{|c|}{ CARACTERÍSTICAS FORMALES } \\
\hline 1 & Número de unidad de análisis & \\
\hline 2 & Fecha & $\begin{array}{l}\text { 1. Septiembre, } 2019 \\
\text { 2. Octubre, } 2019 \\
\text { 3. Noviembre, } 2019 \\
\text { 4. Diciembre, } 2019 \\
\text { 5. Enero, } 2019\end{array}$ \\
\hline 3 & Tipo de unidad de análisis & $\begin{array}{l}\text { 1. Fotografía } \\
\text { 2. Vídeo } \\
\text { 3. Portada de una galería } \\
\text { 4. Contenido interno de una galería }\end{array}$ \\
\hline 4 & Autor de la unidad de análisis & $\begin{array}{l}\text { 1. Gucci } \\
\text { 2. Otro }\end{array}$ \\
\hline \multicolumn{3}{|c|}{ ASOCIACIONES DE MARCA } \\
\hline \multicolumn{3}{|c|}{ Asociaciones con personas } \\
\hline 5 & Celebridades en la unidad de análisis & $\begin{array}{l}\text { 1. Sí } \\
\text { 2. No }\end{array}$ \\
\hline 6 & Profesión de la celebridad & \begin{tabular}{|l|} 
1. Influencer \\
2. Actor \\
3. Músico \\
4. Diseñador \\
5. Varios de los anteriores \\
6. Otros \\
\end{tabular} \\
\hline 7 & Mención a Alessandro Michele & $\begin{array}{l}\text { 1. Sí } \\
\text { 2. No }\end{array}$ \\
\hline
\end{tabular}




\begin{tabular}{|c|c|c|}
\hline 8 & ¿Cómo se mencionó a Alessandro Michele? & $\begin{array}{l}\text { 1. Cuenta } \\
\text { 2. Hashtag } \\
\text { 3. Etiquetado en la imagen } \\
\text { 4. Varias de las anteriores }\end{array}$ \\
\hline 9 & ¿Aparecen modelos? & $\begin{array}{l}\text { 1. Sí } \\
\text { 2. No }\end{array}$ \\
\hline 10 & ¿Cuántos modelos aparecen? & $\begin{array}{l}\text { 1. Uno } \\
\text { 2. Varios }\end{array}$ \\
\hline 11 & Si aparece un único modelo, ¿cuál es su sexo? & $\begin{array}{l}\text { 1. Masculino } \\
\text { 2. Femenino } \\
\text { 3. Indefinido }\end{array}$ \\
\hline 12 & Si aparece un único modelo, ¿cuál es su edad? & $\begin{array}{l}\text { 1. Menor ( } 0 \text { - } 17 \text { años) } \\
\text { 2. Joven ( } 18 \text { - } 30 \text { años) } \\
\text { 3. Adulto (mayores de } 30 \text { años) } \\
\text { 4. No se aprecia }\end{array}$ \\
\hline 13 & Si aparece un único modelo, ¿cuál es su fisionomía? & $\begin{array}{l}\text { 1. Complexión delgada } \\
\text { 2. Complexión media } \\
\text { 3. Complexión gruesa } \\
\text { 4. No se aprecia }\end{array}$ \\
\hline 14 & Si aparece un único modelo, ¿cuál es su raza? & $\begin{array}{l}\text { 1. Asiática } \\
\text { 2. Blanca } \\
\text { 3. Negra } \\
\text { 4. Otros } \\
\text { 5. No se aprecia }\end{array}$ \\
\hline 15 & Si aparecen varios modelos, ¿hay diversidad de sexos? & $\begin{array}{l}\text { 1. Sí } \\
\text { 2. No } \\
\text { 3. No se aprecia }\end{array}$ \\
\hline 16 & Si aparecen varios modelos, ¿hay diversidad de edades? & $\begin{array}{l}\text { 1. Sí } \\
\text { 2. No } \\
\text { 3. No se aprecia }\end{array}$ \\
\hline 17 & Si aparecen varios modelos, ¿hay diversidad de tallas? & $\begin{array}{l}\text { 1. Sí } \\
\text { 2. No } \\
\text { 3. No se aprecia }\end{array}$ \\
\hline 18 & Si aparecen varios modelos, ¿hay diversidad racial? & $\begin{array}{l}\text { 1. Sí } \\
\text { 2. No } \\
\text { 3. No se aprecia }\end{array}$ \\
\hline
\end{tabular}




\begin{tabular}{|c|c|c|}
\hline \multicolumn{3}{|c|}{ Asociaciones con productos } \\
\hline 19 & ¿Aparecen productos de la marca identificables? & $\begin{array}{l}\text { 1. Sí } \\
\text { 2. No }\end{array}$ \\
\hline 20 & Categoría de los productos que aparecen & $\begin{array}{l}\text { 1. Ropa } \\
\text { 2. Complementos } \\
\text { 3. Cosméticos } \\
\text { 4. Perfumes } \\
\text { 5. Calzado } \\
\text { 6. Otros }\end{array}$ \\
\hline \multicolumn{3}{|c|}{ Asociaciones con la organización } \\
\hline 21 & $\begin{array}{l}\text { ¿Se hace referencia a temas relacionados con la Responsabili- } \\
\text { dad Social Corporativa? }\end{array}$ & $\begin{array}{l}\text { 1. Sí } \\
\text { 2. No }\end{array}$ \\
\hline 22 & ¿Se hace referencia al Grupo Kering? & $\begin{array}{l}\text { 1. Sí } \\
\text { 2. No }\end{array}$ \\
\hline \multicolumn{3}{|c|}{ Asociaciones con símbolos } \\
\hline 23 & ¿Aparece algún elemento icónico reconocible de la marca? & $\begin{array}{l}\text { 1. Logotipo de Gucci sobreimpreso } \\
\text { 2. Logotipo completo de Gucci } \\
\text { 3. Monograma doble G } \\
\text { 4. Estampado monogram } \\
\text { 5. Franja bicolor Gucci } \\
\text { 6. Hebillas horsebit } \\
\text { 7. Serpiente } \\
\text { 8. Libélula } \\
\text { 9. Otros } \\
\text { 10. Varios de los anteriores } \\
\text { 11. Ninguno }\end{array}$ \\
\hline
\end{tabular}

Fuente: elaboración propia

\section{Resultados}

Durante el periodo que abarca nuestra investigación -de septiembre de 2019 a enero de 2020- contabilizamos un total de 642 publicaciones en la cuenta Gucci Official en Instagram. En el presente apartado se expondrán los resultados obtenidos del análisis de todas ellas, mostrando en primer lugar las características formales y, posteriormente, las de contenido.

\subsection{Características formales de las publicaciones}

Según se muestra en la tabla 3, octubre es el mes que mayor porcentaje de publicaciones registró (33\%). En noviembre $(23,8 \%)$ y diciembre $(23,1 \%)$ desciende el volumen de publicaciones, sin encontrar apenas diferencias entre ellos. Septiembre (10\%) y enero $(10,1 \%)$ fueron los meses con menor frecuencia de publicación, aunque estas cifras son muy relativas al no haber estudiado estos meses en su totalidad, ya que se partió del 27 de septiembre y se terminó el 12 de enero. 
Tabla 3. Periodo de la publicación

\begin{tabular}{|l|l|}
\hline Mes de la publicación & Porcentaje \\
\hline Septiembre de 2019 & 10 \\
\hline Octubre de 2019 & 33 \\
\hline Noviembre de 2019 & 23,8 \\
\hline Diciembre de 2019 & 23,1 \\
\hline Enero de 2020 & 10,1 \\
\hline Total & 100 \\
\hline
\end{tabular}

Fuente: elaboración propia

Casi la mitad de las publicaciones forman parte del contenido interno de las galerías (49,5\%). No obstante, si consideramos cada galería como una unidad independiente, sus portadas representan el 15\% de las publicaciones. Asimismo, las fotografías individuales suponen un 28,5\% de los contenidos compartidos por la firma y, por último, los vídeos publicados de manera individual completan el $7 \%$ restante.

Respecto a la autoría de las publicaciones, entendemos como autor aquella cuenta de Instagram que realizó la publicación original para así, poder diferenciar entre las publicaciones firmadas por Gucci y aquellas de otras cuentas que fueron reposteadas por la marca.

Los resultados sitúan a Gucci como la única autora de la totalidad del contenido de la firma. Es decir, el $100 \%$ de los contenidos que publicó Gucci fueron creados por la marca, no utilizando contenido de otras cuentas con la opción de repost.

\subsection{Asociaciones de la marca con personas}

El estudio de la comunicación de la identidad de la marca Gucci en Instagram se ha estructurado en torno a las cuatro perspectivas de construcción de la identidad de marca que plantea Aaker (1996): persona, producto, organización y símbolo.

En primer lugar, desarrollamos la perspectiva de la marca como persona a través de las asociaciones que establece Gucci con determinadas personas en su perfil de Instagram.

En el 23,2 \% de las publicaciones de la marca aparece alguna celebridad, entendiendo como tales aquellas personas con fama y notoriedad social que no están ejerciendo como modelos en el momento de la publicación. Dado que el celebrity endorsement es una práctica habitual en la estrategia de comunicación de la marca, vamos a ilustrar los datos mostrados en la tabla 4 con algunos ejemplos. 
La mayoría de estas celebridades son actores (40,3\%) y músicos (26,2\%). Un ejemplo de los primeros es Gwyneth Paltrow, quien aparece en contextos diversos tales como un fotograma de la serie The Politician, la portada de la revista Harper's Bazaar o un robado de la actriz en Nueva York. En todas las publicaciones, viste alguna prenda de Gucci a la que se hace referencia explícita. Las actrices Sienna Miller y Elle Fanning aparecen posando en algún evento con prendas de la marca. Además, la primera también ejerce como modelo para Gucci.

Figura 1. Publicación referente a la actriz Gwyneth Paltrow

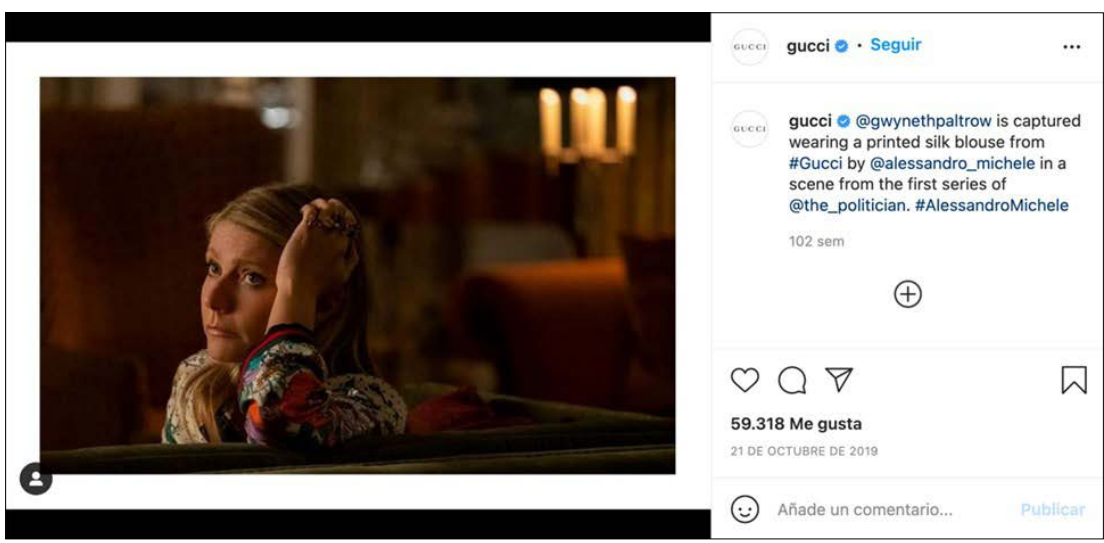

Fuente: Gucci Official (2019)

Entre los músicos destaca Iggy Pop, quien aparece en un reportaje de la revista GQ con una camisa Gucci o actuando en un evento organizado por la marca. Asimismo, Harry Styles es mostrado en la escena de un videoclip o actuando en televisión con prendas de Gucci. Ambos músicos también ejercen como modelos para la marca.

La presencia de influencers es muy escasa (2\%), dato que confirma la independencia de la marca respecto a estas figuras. En este caso, destacamos la vinculación con Sinead Burke, activista por el diseño inclusivo en la industria de la moda, algo que refuerza la apuesta de la marca por la diversidad.

Las celebridades provenientes de otros ámbitos (20,8\%) suelen estar vinculadas con el arte y la cultura. Un ejemplo es la publicación dedicada al cineasta Alfonso Cuarón, quien recibió un reconocimiento en la Gala LACMA Art + Film patrocinada por Gucci. En este sentido, Velar-Lera et al. (2020) destacan el frecuente uso del arte como elemento narrativo por parte de la firma italiana. 
Tabla 4. Profesión de las celebridades

\begin{tabular}{|l|l|}
\hline Profesión de las celebridades & Porcentaje \\
\hline Actor & 40,3 \\
\hline Músico & 26,2 \\
\hline Influencer & 2 \\
\hline Diseñador & 1,3 \\
\hline Varios de los anteriores & 9,4 \\
\hline Otros & 20,8 \\
\hline Total & 100 \\
\hline
\end{tabular}

Fuente: elaboración propia

Sin embargo, el mayor protagonismo lo obtiene Alessandro Michele, quien aparece mencionado en casi la totalidad de las publicaciones $(97,5 \%)$ mediante etiquetas en la imagen, menciones a su cuenta o hashtags. Además, en la mayor parte de las ocasiones $(98,1 \%)$, se hace uso de varias de estas herramientas en una misma publicación. Observamos que el vínculo de la marca con su director creativo es muy estrecho al mencionarlo incluso en aquellas publicaciones en las que no aparece su imagen. Este dato confirma el rol del director creativo como influencer de la propia marca (Pérez-Curiel y Sanz-Marcos, 2019).

Respecto a la presencia de modelos, estos aparecen en el 55,9\% de las publicaciones. A este respecto, es más común encontrar publicaciones con un único modelo (59,6\%) que con varios (40,4\%). A continuación, se muestran los resultados correspondientes a las principales características de los modelos en aquellas publicaciones en las que aparece un único modelo.

En el 36,8\% de las publicaciones, los modelos que aparecen son mujeres, mientras que en el 27,9\%, son hombres. En el 35,3\% restante, no se ha podido diferenciar con claridad esta característica debido a la pose, a la ambigüedad del modelo o al encuadre de la imagen.

La edad de los modelos se sitúa en la mayor parte de las publicaciones entre los 18 y los 30 años (42,5\%). Los modelos cuya edad aparenta ser superior a los 30 años aparecen en un 18,7 \% de las publicaciones. A este respecto, cabe señalar que la asignación de la edad quedó a interpretación del investigador, al no ser posible comprobarla con exactitud en todos los casos.

En esta ocasión, también encontramos un amplio volumen de publicaciones en las que no se logra apreciar este rasgo (38,3\%), debido a los mismos motivos que se apuntaban anteriormente. Por último, la presencia de menores de edad es prácticamente inexistente $(0,5 \%)$.

El predominio de modelos con apariencia juvenil es coherente con la orientación de la marca a un público más joven. No obstante, la destacable presencia de modelos bastante más maduros pone de manifiesto el eclecticismo y la diversidad que caracterizan a la marca, tal como se muestra en la figura 2 . 
Figura 2. Publicación referente a la Colección Crucero 2020 de Gucci

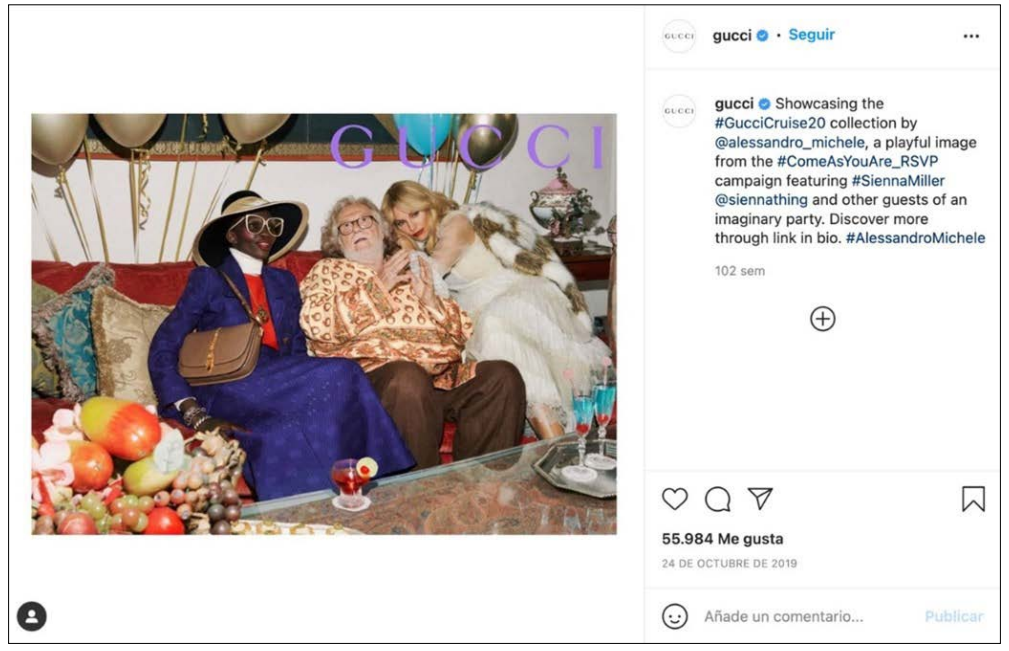

Fuente: Gucci Official (2019)

Con respecto a la complexión física del modelo, en más de la mitad de las publicaciones en las que aparece un único modelo, este tiene una complexión delgada $(55,6 \%)$ o media $(11,3 \%)$. De nuevo, hay un amplio porcentaje de publicaciones en las que no se puede valorar esta característica $(32,2 \%)$, al no apreciarse la silueta del modelo por diversas razones. Por último, es destacable la escasa presencia de modelos con complexión gruesa (0,9\%). Observamos que el eclecticismo y diversidad con los que la marca se identifica no quedan reflejados en este aspecto.

En las publicaciones con un único modelo, la raza con mayor representatividad es la blanca (41,1\%), seguida a bastante distancia por la negra $(13,9 \%)$ y la asiática $(11,5 \%)$, según se observa en tabla 5 . En esta ocasión, también encontramos un abundante número de publicaciones en los que no se logra apreciar esta característica (32,1\%), por los motivos expuestos con anterioridad.

Tabla 5. Raza del modelo

\begin{tabular}{|c|c|}
\hline Raza de los modelos & Porcentaje \\
\hline Asiática & 11,5 \\
\hline Blanca & 41,1 \\
\hline Negra & 13,9 \\
\hline Otros & 1,4 \\
\hline No se aprecia & 32,1 \\
\hline Total & 100 \\
\hline
\end{tabular}

Fuente: elaboración propia 
Seguidamente, se muestran los resultados correspondientes a las publicaciones en las que aparecen varios los modelos, algo que aportará más información acerca de la diversidad que Gucci muestra en su perfil de Instagram.

En el 51,7\% de las publicaciones se observa diversidad de sexos. En el 21,4\% de los casos solo aparece un sexo representado y en el 26,9\% restante, no pudo apreciarse esta característica.

La diversidad racial también es mayoritaria, al estar presente en el 51\% de los casos, frente al 23,5\% en los que aparecen modelos de una única raza. Por último, en el 25,5\% de los casos, tampoco pudo apreciarse esta característica.

Es igualmente reseñable la diversidad de edades observada en el $40 \%$ de las publicaciones, frente al 28,3\%, donde la edad de los modelos es homogénea y el 31,7\% en el que no se pudo apreciar.

Al comparar estos resultados con los obtenidos anteriormente en publicaciones con un único modelo, observamos que la diversidad de razas y edades tiene mayor representación en aquellas publicaciones en las que aparecen varios modelos.

La talla de los modelos es la única variable en la que la homogeneidad (39,3\%) es superior a la diversidad (36,6\%). Cabe apuntar que, en todos estos casos, la representación de tallas grandes es prácticamente inexistente, con un predominio de complexiones delgadas y medias.

\subsection{Asociaciones de la marca con productos}

Los productos de la marca aparecen en el 55\% de las publicaciones analizadas. Este resultado confirmaría la importancia que Gucci concede al producto en su estrategia de comunicación en Instagram (Pérez-Curiel y Sanz-Marcos, 2019).

En la tabla 6 observamos el predominio de los complementos (47,9\%), seguidos por la ropa (34,3\%) y el calzado, cuya presencia es más discreta $(10,2 \%)$. La escasa presencia de perfumes $(2,8 \%)$ y cosméticos $(3,1 \%)$ puede deberse a que la firma tiene una cuenta específica para el sector de la belleza.

Tabla 6. Categoría de producto

\begin{tabular}{|l|l|}
\hline Categoría de producto & Porcentaje \\
\hline Ropa & 34,3 \\
\hline Complementos & 47,9 \\
\hline Cosméticos & 3,1 \\
\hline Perfumes & 2,8 \\
\hline Calzado & 10,2 \\
\hline Otros & 1,7 \\
\hline Total & 100 \\
\hline
\end{tabular}

Fuente: elaboración propia 


\subsection{Asociaciones de la marca con la organización}

En la perspectiva de la marca como organización, se han registrado las publicaciones que tratan sobre temas de RSC y las que hacen referencia al Grupo Kering.

Tan solo el 3,3\% de las publicaciones hacen referencia a temas relacionados con la responsabilidad social corporativa. Destacan aquellas referentes al programa de becas Gucci Changemakers, dirigidas a estudiantes norteamericanos con interés en el mundo de la moda (Gucci Equilibrium, 2021).

Figura 3. Publicación referente a una acción de RSC
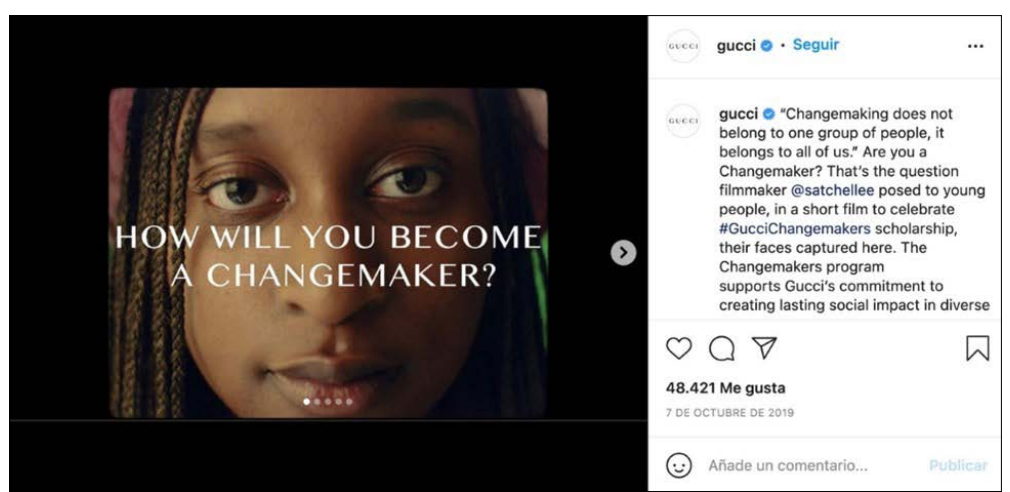

Fuente: Gucci Official (2019)

Es destacable la ausencia de vínculos entre la firma italiana y el Grupo Kering, dado que en ninguna de las publicaciones estudiadas existen referencias al grupo empresarial francés $(0 \%)$.

\subsection{Asociaciones de la marca con símbolos}

En este último apartado se analiza la presencia de representaciones simbólicas de la marca en su perfil de Instagram a través de sus iconos más representativos.

En el 52\% de las publicaciones, aparece al menos un elemento icónico de la firma. Lo más frecuente es que aparezcan varios de ellos en una misma publicación (31,2\%) plasmados en diferentes prendas y/o complementos, según se observa en la tabla 7 .

En las publicaciones en las que aparece un único elemento, el más utilizado es el estampado monogram (19,8\%). En segundo lugar, se encuentran las hebillas horsebit (11,9\%), seguidas del monograma de la doble G (9,6\%).

El logotipo completo de Gucci aparece plasmado sobre alguna prenda de vestir en el 7,7\% de las publicaciones. Además, en el 8,5\% de los casos se ha añadido posteriormente y aparece sobreimpreso en primer plano.

La franja bicolor de Gucci aparece con menor frecuencia (3,7\%). No obstante, en varias ocasiones ocupa más espacio que otros iconos de la firma, al actuar como fondo de la publicación. 
Los elementos icónicos menos representados fueron la serpiente (0,4\%) y las libélulas (0,4\%) que Alessandro Michele introdujo en el imaginario de la firma. Observamos, por tanto, que los iconos más utilizados son los que han acompañado a la marca durante más tiempo.

Tabla 7. Presencia de elementos icónicos

\begin{tabular}{|l|l|}
\hline Elemento icónico & Porcentaje \\
\hline Logotipo de Gucci sobreimpreso & 8,5 \\
\hline Logotipo completo de Gucci & 7,7 \\
\hline Monograma doble G & 9,6 \\
\hline Estampado monogram & 19,8 \\
\hline Franja bicolor Gucci & 3,7 \\
\hline Hebillas horsebit & 11,9 \\
\hline Serpiente & 0,4 \\
\hline Libélula & 0,4 \\
\hline Otros & 6,8 \\
\hline Varios de los anteriores & 31,2 \\
\hline Total & 100 \\
\hline
\end{tabular}

Fuente: elaboración propia

\section{Discusión y conclusiones}

El carácter visual e interactivo de Instagram y las diversas funcionalidades que integra la aplicación, permiten a las marcas de moda generar un mayor engagement con los usuarios. Esto supone una oportunidad para afianzar su relación con las nuevas generaciones de consumidores. Sin embargo, también plantea un desafío para las marcas del sector del lujo, al tener que adaptar su comunicación al entorno digital manteniendo intacta la percepción de exclusividad.

Partiendo de este marco, el estudio realizado muestra como Gucci ha adaptado su estrategia de comunicación a Instagram, permaneciendo fiel a sus principales atributos de identidad.

En primer lugar, la totalidad de las publicaciones fueron creadas por la firma y no tomadas de otros perfiles de Instagram. Este control sobre la autoría de los contenidos refuerza la percepción de exclusividad e independencia de la marca. Gucci crea tendencias en lugar de seguirlas y se sitúa como una marca influyente desde su cuenta de Instagram.

Gucci se vincula a celebridades en aproximadamente una de cada cuatro publicaciones, recurriendo a diversas fórmulas de celebrity endorsement. Aunque sus perfiles son heterogéneos, cada una de ellas encarna alguno de los atributos con los que la 
marca se identifica. Un ejemplo es la vinculación con el cantante Iggy Pop, conocido por su actitud transgresora e irreverente. El predominio de actores, músicos y otras personalidades relacionadas con el arte y la cultura es coherente con el posicionamiento creativo y vanguardista de la marca. Algunas de estas celebridades están muy integradas en la comunicación de la marca e incluso desempeñan el rol de modelo, una práctica cada vez más habitual en el ámbito de la moda de lujo como señala Barron (2021). En cambio, el protagonismo concedido a los influencers es prácticamente inexistente, lo que confirma el carácter independiente de la marca, cuya capacidad de influencia no depende de agentes externos (Pérez-Curiel y Sanz-Marcos, 2019). Al comparar este resultado con los obtenidos por Velar-Lera et al. (2020), observamos que hay marcas como Saint Laurent, Burberry o Chanel que recurren a estas figuras con una frecuencia muy superior a Gucci, algo que reafirma la independencia de la marca.

Sin embargo, el vínculo más estrecho es el formado con el director creativo, Alessandro Michele. La figura del diseñador es omnipresente en el contenido de la marca, ya que su nombre aparece en prácticamente todas las publicaciones, ya sea en forma de hashtag o de mención. En la investigación llevada a cabo por Velar-Lera et al. (2020), se observa que, para el conjunto de las marcas estudiadas, las menciones al director creativo representan una frecuencia muy inferior (6,6\%). Esto pone aún más de manifiesto la importancia de Alessandro Michele en la estrategia de comunicación digital de Gucci, al posicionarse como principal influencer de la marca (Pérez-Curiel y Sanz-Marcos, 2019). Podemos concluir, por tanto, que Alessandro Michele constituye la principal seña de identidad de Gucci en el presente y que su figura encarna los principales atributos con los que se identifica la marca.

Estas conclusiones demuestran la validez de la primera hipótesis, en la que adelantábamos que la figura de Alessandro Michele ocupa un lugar preferente en las publicaciones de la marca respecto a otras celebridades e influencers, cuyo protagonismo es inferior.

El eclecticismo y la modernidad con los que se identifica la firma italiana están representados en la mayor parte de las características de los modelos que ocupan sus publicaciones. La diversidad racial y de edades se observa especialmente en aquellas publicaciones en las que aparecen varios modelos.

Como se ha comentado anteriormente, destaca la importancia que Gucci concede a las celebridades y su tendencia a incluirlas como modelos de sus colecciones. Un rasgo de identidad característico de la marca es la combinación de personas anónimas con celebridades, acentuando de este modo su carácter ecléctico y heterogéneo.

La diversidad de sexos también es frecuente en sus publicaciones, así como la ambigüedad de algunos de los modelos. Esto guarda relación con el cambio impulsado por Gucci desde 2016, a partir del cual, la firma decide presentar sus colecciones de mujer y hombre de manera simultánea en un único desfile. De este modo, la marca se desvincula del concepto binario de género y se sitúa en una posición más vanguardista e integradora.

Sin embargo, el carácter inclusivo de la marca no queda reflejado en la diversidad de tallas. En prácticamente ninguna publicación aparecen representadas las tallas grandes, y las pocas ocasiones en que esto sucede, se vinculan a acciones de RSC.

Por lo tanto, comprobamos que, mientras que la variedad de sexos, razas y edades está ampliamente representada en las publicaciones analizadas, el límite de la diversidad de Gucci lo marca la complexión corporal. 
De este modo se confirma parcialmente nuestra segunda hipótesis: el eclecticismo de la marca no solo queda reflejado en sus diseños, sino también en la diversidad y características físicas de sus modelos.

La cuenta oficial de Gucci en Instagram es un escaparate que permite a la firma dar a conocer sus novedades y renovarse continuamente. Así, en más de la mitad de sus publicaciones, aparecen productos identificables de la marca. Esto confirma la absoluta identificación de Gucci con sus productos, que se sitúan como eje central de comunicación y principal activo de la marca (Pérez-Curiel y Sanz-Marcos, 2019).

La amplia presencia de complementos refuerza la asociación de la marca con esta categoría de productos, vinculados a los orígenes artesanales de la firma. Además, las cuidadas fotografías de producto ensalzan la riqueza de los materiales y la meticulosidad de los detalles, potenciando la percepción de lujo, calidad y exclusividad inherentes a la marca.

Los diseños de Alessandro Michele se caracterizan por la reinterpretación y combinación de estilos provenientes de diversas épocas y culturas. De este modo, el producto es un reflejo de eclecticismo y romanticismo de su autor.

Todo lo anterior confirma la tercera hipótesis, en la que se anticipaba la elevada vinculación de la marca con sus productos en los contenidos de sus publicaciones.

Por otra parte, Gucci no hace ninguna mención al Grupo Kering en su contenido de Instagram durante el periodo analizado. Al desvincularse de la marca matriz, Gucci preserva su identidad, mostrándose de nuevo como una firma independiente.

Los contenidos relacionados con la responsabilidad social corporativa son prácticamente inexistentes. Sin embargo, esto no significa que Gucci no tenga interés en mostrarse como una marca socialmente responsable. En su plataforma Gucci Equilibrium (equilibrium.gucci.com), la firma manifiesta su compromiso con diversas causas sociales, entre las que destacan la lucha por la igualdad de género y la sostenibilidad. Esta iniciativa tiene su propia cuenta de Instagram, donde se publican contenidos específicos sobre responsabilidad social de la marca.

Aunque Gucci no comunique explícitamente este tipo de contenidos en su cuenta oficial de Instagram, algunos de los valores de la marca quedan patentes en sus publicaciones, especialmente los relacionados con la igualdad de género, la diversidad y la inclusión, por las razones que se han apuntado anteriormente. De este modo, la marca se muestra sensible hacia determinadas causas sociales.

Respecto a la dimensión icónica de la marca, en algo más de la mitad de las publicaciones aparece, al menos, uno de sus símbolos. En la mayor parte de los casos, se muestran de forma discreta, integrados en el diseño de ciertos productos. En algunas publicaciones, el logotipo de Gucci adquiere una mayor presencia al aparecer sobreimpreso en grandes dimensiones sobre la imagen.

La iconografía clásica de la marca es la que más protagonismo obtiene. El monograma de la doble G es el símbolo más repetido, especialmente en su aplicación en el estampado monogram. Esto evidencia el orgullo que Alessandro Michele afirma sentir por este icono. Por otra parte, los nuevos símbolos introducidos por el director creativo tienen una presencia mucho más discreta. La simbología clásica de Gucci nos traslada a la dimensión más mítica de la marca y pone de manifiesto su romanticismo al evocar su pasado.

A partir de todas las conclusiones anteriores, podemos confirmar la cuarta y principal hipótesis de esta investigación: la comunicación de Gucci en Instagram es coherente con los principales atributos de identidad expresados por la marca. 
La principal aportación respecto a investigaciones precedentes es la reflexión acerca de los atributos de identidad de la marca Gucci y de qué modo se plasman en su comunicación en Instagram. Particularmente, este trabajo confirma algunos de los resultados obtenidos por Pérez-Curiel y Sanz-Marcos (2019) y los amplía con otras aportaciones. Asimismo, el estudio en profundidad de una única marca complementa la investigación de Velar-Lera et al. (2020), cuya perspectiva es más general al abarcar la actividad de varias marcas de lujo en Instagram.

El análisis de contenido, estructurado según el modelo de identidad de marca de Aaker (1996), presenta un enfoque novedoso que permite ser replicado en futuras vías de investigación. De este modo, se podrían completar los resultados obtenidos ampliando la muestra cronológica, extrapolando el código a otras marcas de moda o estudiando la comunicación de Gucci en otros medios.

\section{Agradecimientos}

La traducción al inglés de este artículo ha sido realizada por Charles E. Arthur.

\section{Referencias bibliográficas}

Aaker, D. (1996). Construir marcas poderosas. Gestión 2000.

Alonso, G. y Arébalos A. (2011). La Revolución Horizontal. LibrosEnRed. https://bit.ly/3ar342N

Amed, I. (20 de marzo de 2015). Inside Balmain's digital revolution. Business of Fashion. https://bit.ly/3DxKylJ

Ávila, L. (2017). Instagram para todos. Hablando en corto. https://bit.ly/3Dp7S5c

Barron, L. (2021). The Return of the Celebrity Fashion Muse: Brand Endorsement, Creative Inspiration and Celebrity-Influenced Design Communication. Fashion Theory, 25:6, 757-776. https://doi.org/10.1080/1362704X.2019.1656946

Berelson, B. (1952). Content Analysis in communication research. Free Press.

Campi i Valls, I. (2007). Diseño y nostalgia. El consumo de la historia. Santa \& Cole.

Capriotti, P. (2009). Branding Corporativo. Fundamentos para la gestión estratégica de la Identidad Corporativa. Business School Universidad Mayor.

Carrillat, F. A., e Ilicic, J. (2019). The Celebrity Capital Life Cycle: A Framework for Future Research Directions on Celebrity Endorsement. Journal of Advertising, 48(1), 61-71. https://doi.org/10.1080/00913367.2019.1579689

Deloitte. (2020). Global powers of luxury goods 2020: The new age offashion and luxury. https://bit.ly/2YzZFMr

Deloitte (2019). Las Potencias Globales de Artículos de Lujo 2019. https://bit.ly/3ycrFDf

Dessart, L., Veloutsou, C., y Morgan-Thomas, A. (2015). Consumer engagement in online brand communities: a social media perspective. Journal of Product \& Brand Management, 24(1), 28-42. https://doi.org/10.1108/JPBM-06-2014-0635

Driessens, O. (2013). Celebrity capital: redefining celebrity using field theory. Theory and Society, 42(5), 543-560. https://doi. org/10.1007/s11186-013-9202-3 
Estévez-Martín, A., Izquierdo-González, V., y Soler-Guirao, B. (2019). La moda de lujo en las redes sociales: Análisis del uso de Instagram por parte de la marca de moda de lujo Loewe. Creatividad y Sociedad (extraordinario), 142-179. https://bit.ly/31odIpT Fernández-Gómez, J. D., Hernández-Santaolalla, V., y Sanz-Marcos, P. (2018). Influencers, marca personal e ideología política en Twitter. Cuadernos.Info, (42), 19-37. http://dx.doi.org/10.7764/cdi.42.1348

Gautier, J. (2011). Chanel. Las claves de un estilo. La Fábrica.

Girón, M. E. (2011). Secretos de lujo. LID Editorial.

Gucci. (s.f.). Acerca de Gucci. https://bit.ly/3yarW9N

Gucci Equilibrium. (2021). Programas de becas en Norteamérica de Gucci Changemakers. https://bit.ly/3FKM8Tw

Gucci Official [@gucci]. (21 de octubre de 2019). @gwynethpaltrow is captured wearing a printed silk blouse from \#Gucci by @ alessandro_michele in a scene from the first series of @the_politician [Fotografía]. Instagram. https://bit.ly/3Dvwycw

Gucci Official [@gucci]. (24 de octubre de 2019). Showcasing the \#GucciCruise20 collection by @alessandro_michele [Fotografía]. Instagram. https://bit.ly/3AuD69o

Gucci Official [@gucci]. (7 de octubre de 2019). "Changemaking does not belong to one group of people, it belongs to all of us.” [Fotografía]. Instagram. https://bit.ly/3oO3oBd

Iab Spain. (2021). Estudio de Redes Sociales 2021. https://bit.ly/30gSvO4

Iab Spain. (2019). Libro Blanco Marketing de Influencers. https://bit.ly/2YCUw6c

Interbrand. (2020). Best Global Brands. https://bit.ly/33JO2BZ

Jackson, T., y Haid, C. (2002). Gucci Group - The New Family of Luxury Brands. International Journal of New Product Development and Innovation Mangement, 4, 161-172. https://bit.ly/3opi0EP

Kerlinger, F. (1986). Foundations of behavioural research. Praeger.

Kim, A. J., y Ko, E. (2012). Do Social Media Marketing Activities Enhance Customer Equity? An Empirical Study of Luxury Fashion Brand. Journal of Business Research, 65 (10), 1480-1486. https://doi.org/10.1016/j.jbusres.2011.10.014

Ko, E., Phau, I., y Aiello, G. (2016). Luxury brand strategies and customer experiences: Contributions to theory and practice. Journal of Business Research, 69(12), 5749-5752. https://doi.org/10.1016/j.jbusres.2016.04.170

Liberos, E., Núñez A., Bareño R., García del Poyo, R., Gutiérrez J., y Pino, G. (2013). El libro del marketing interactivo y la publicidad digital. ESIC Editorial.

Llamas, F., y Pagador, I. (2014). Estudio sobre las redes sociales y su implicación en la adolescencia. Enseñanza \& Teaching Revista Interuniversitaria De Didáctica, 32(1), 43-57. https://doi.org/10.14201/et20143214357

Martínez, J. (12 de febrero de 2020). Kering dispara sus ingresos un 13 por ciento y supera la barrera de los 15 mil millones. Fashion United. https://bit.ly/3uNIF0o

Moreno, M. (2018). La enciclopedia del Community Manager. Ediciones Deusto. 
Okonkwo, U. (2009). The luxury brand strategy challenge. Journal of Brand Management, 16(5-6), 287-289. https://doi. org/10.1057/bm.2008.53

Okonkwo, U. (2007). Luxury fashion branding. Trends, tactics and tecniques. Palgrave Macmillan.

Oliveira, M., y Fernandes, S. (2020). Luxury brands and social media: drivers and outcomes of consumer engagement on Instagram. Journal of Strategic Marketing. https://doi.org/10.1080/0965254X.2020.1777459

Park, M., Im, H., y Kim, H. (2019). "You are too friendly!" The negative effects of social media marketing on value perceptions of luxury fashion brands. Journal of Business Research, 117, 529-542. https://doi.org/10.1016/j.jbusres.2018.07.026

Pentina, I., Guilloux, V., y Micu, A. C. (2018). Exploring Social Media Engagement Behaviors in the Context of Luxury Brands. Journal of Advertising, 47(1), 55-69. https://doi.org/10.1080/00913367.2017.1405756

Pérez-Curiel, C., y Sanz-Marcos, P. (2019). Estrategia de marca, influencers y nuevos públicos en la comunicación de moda y lujo. Tendencia Gucci en Instagram. Revista Prisma Social, (24), 1-24. https://bit.ly/3EH5WWx

Sanz-Marcos, P., y Pérez-Curiel, C. (2019). Branding e influencers en la comunicación de moda. Gucci como caso de estudio. Redmarka. Revista de Marketing Aplicado, 23(1), 21-39. https://doi.org/10.17979/redma.2019.23.1.5452

Sierra, R. (1997). Técnicas de investigación social. Teoría y ejercicios. Thomson.

Socialbakers. (2021). Estado de las Redes Sociales y el CX Q1 2021. https://bit.ly/3mF9Qrv

Statista. (2021a). Distribution of Instagram users worldwide as of July 2021, by age group. https://bit.ly/3v2kvQX

Statista. (2021b). Number of Instagram users worldwide from 2016 to 2023. https://bit.ly/3FAmlxb

Statista. (2021c). Distribution of Instagram users worldwide as of July 2021, by gender. https://bit.ly/3aGQ3T7

The Fashion Law (23 de agosto de 2016). With New Iconographyat Play, Gucci is Banking on LegalProtection. https://bit.ly/3tIZ5WJ The Lyst Index. (2020). The Lyst Index 2T 2020. https://bit.ly/3tPf0Ta

Velar-Lera, M., Herrera-Damas, P., y González-Aldea, P. (2020). Uso de Instagram para la comunicación de las marcas de moda de lujo. Palabra Clave, 23(4), e2344. https://doi.org/10.5294/pacla.2020.23.4.4

Vicente-Fernández, P., Vinader-Segura, R., y Soria-Ibáñez, M.M. (2020). Redes sociales al servicio del marketing: análisis comparativo en el caso de Dulceida. Revista Espacios, 41(24), 342-358.

Vogue. (s.f.). Gucci. https://bit.ly/33K6RVz

Wimmer, R., y Dominick, J. (1996). La investigación científica de los medios de comunicación. Una introducción a sus métodos. Bosch Comunicación.

Yoffie, D., y Kwak, M. (2001). Gucci Group N.V. (A). Harvard Business School Case, 703-S10. https://bit.ly/3hsc7Ft

Zurutuza, C. (2012). Técnicas de investigación social en comunicación. En Zugasti, R. (Coord.). Investigar en ciencias sociales: el estudio de la comunicación (pp. 101-121). Ediciones Universidad San Jorge. 